\title{
CONTINUOUS SINGULAR MEASURES WITH ABSOLUTELY CONTINUOUS CONVOLUTION SQUARES
}

\author{
ANTHONY H. DOOLEY AND SANJIV KUMAR GUPTA
}

(Communicated by Palle E. T. Jorgensen)

\begin{abstract}
We prove for every non-abelian compact connected group $G$ there is a continuous, singular, central measure $\mu$ with $\mu * \mu$ in $L^{p}$ for all $p, 1 \leq p<$ $\infty$. We also construct such measures on some families of non-abelian compact totally disconnected groups. These results settle an open question of Ragozin.
\end{abstract}

\section{INTRODUCTION}

In 1966, Hewitt and Zuckerman [6] proved that if $G$ is a non-discrete locally compact abelian group with Haar measure $\lambda$, then there exists a non-negative, continuous, regular measure $\mu$ on $G$ that is singular to $\lambda(\mu \perp \lambda)$ such that $\mu(G)=$ $1, \mu * \mu$ is absolutely continuous with respect to $\lambda(\mu * \mu<<\lambda)$ and the LebesgueRadon-Nikodym derivative of $\mu * \mu$ with respect to $\lambda$ is in $L^{p}(G, \lambda)$ for all $p, 1 \leq$ $p<\infty$. This result was partially generalised to non-abelian connected compact simple groups by Ragozin [10] and to any locally compact group (nondiscrete) by Karanikas and Koumandos [7]. They showed that there exist continuous singular measures $\mu$ with $\mu * \mu \in L^{1}(G, \lambda), \lambda$ being the left Haar measure on $G$. Ragozin [10] mentioned that even for connected compact simple Lie groups it is an open question whether there exists a singular measure $\mu$ with $\mu * \mu \in L^{p}(G, \lambda)$ for all $p, 1 \leq p<\infty$.

In this paper, we show that on any non-abelian compact, connected group or on any compact Lie group $G$, there exist central, continuous, singular measures $\mu$ with $\mu * \mu \in L^{p}(G, \lambda)$ for all $p, 1 \leq p<\infty$ and also prove this result on some specific disconnected non-abelian compact groups. We also note two remarkable but difficult works of Saeki [11], [12] and a paper of Stempak [14] on this subject.

In section 2, we establish our notation. We state and prove our main theorem (Theorem 3.1) in section 3. Section 4 contains the results on some specific totally disconnected compact groups.

\section{NotATION}

$G$ always denotes a non-abelian compact group unless stated otherwise. Let $G$ be a compact connected Lie group and $T=\mathbb{T}^{\ell}$ be a maximal torus for $G$. Let $R$ denote the set of roots of $G$ with respect to $T, \triangle$ a base for $R$, and $R_{+}$the set of positive roots with respect to $\triangle$.

Received by the editors September 4, 1994 and, in revised form, April 3, 1995.

1991 Mathematics Subject Classification. Primary 43A77.

(C)1996 American Mathematical Society 
The Weyl group $W$ is the finite group $N_{G}(T) / T$, where $N_{G}(T)$ is the normaliser of $T$ in $G$ and one has an action on $T$ given by

$$
w=g T: t \rightarrow w t=g t g^{-1} .
$$

For each $w \in W, H \rightarrow d w(e) H$ defines an action of $w$ on the Lie algebra $\underline{\underline{t}}$ of $T$ which we denote also by $H \rightarrow w H$. Let $\operatorname{sgn}(w)$ denote the determinant of $d w(e)$.

Let $\underline{g}^{*}$ and $\underline{\underline{t}}^{*}$ denote the duals of the real Lie algebras $\underline{g}$ and $\underline{\underline{t}}$ respectively. The derivatives of the roots of $G$ on $T$ are purely imaginary linear forms on $\underline{\underline{t}}$. For $\alpha \in R$, let $d \alpha(e)=i \tilde{\alpha}$ where $\tilde{\alpha} \in \underline{\underline{t}}^{*}$. Let $($,$) denote the Killing form on \underline{\underline{t}}$, and by duality transfer it to a form on $\underline{\underline{t}}^{*}$. For $H_{1}, H_{2} \in \underline{\underline{t}}$, we denote $\frac{2\left(H_{1}, H_{2}\right)}{\left(H_{2}, H_{2}\right)}$ by $\left\langle H_{1}, H_{2}\right\rangle$.

For $p \in \underline{\underline{t}}^{*}$, we denote by $H_{p} \in \underline{\underline{t}}$ the element satisfying

$$
p(H)=\left(H_{p}, H\right) \quad \forall H \in \underline{\underline{t}} .
$$

Let $\triangle=\left\{\alpha_{1}, \cdots, \alpha_{\ell}\right\}$. We denote by $\check{\alpha}_{i}=\frac{2 \tilde{\alpha}_{i}}{\left(\tilde{\alpha}_{i}, \tilde{\alpha}_{i}\right)}, 1 \leq i \leq \ell$.

Let $\delta=1 / 2 \sum_{\alpha \in R_{+}} \tilde{\alpha}$ and $q=\prod_{\alpha \in R_{+}}(1-\bar{\alpha})$. Define $j$, an Ad-invariant function on $\underline{\underline{g}}$ as follows: for $H \in \underline{\underline{t}}$, set

$$
j(H)=\frac{e^{i \delta(H)} q(\exp H)}{\prod_{\alpha \in R_{+}} i \tilde{\alpha}(H)} .
$$

Then $|j|^{2}$ is exactly the determinent of the exponential map, and in fact $j$ takes real values.

Now we define the wrapping map $\Phi[2]$. Let $\mu \in M(\underline{g})$ be an Ad-invariant measure of compact support. Define

$$
\langle\Phi \mu, \varphi\rangle=\langle\mu, j \tilde{\varphi}\rangle \quad \forall \varphi \in C(G)
$$

where

$$
\tilde{\varphi}(X)=\varphi(\exp X)
$$

It follows easily that $\Phi \mu$ is a central measure on $G$ and if $\mu$ is continuous then $\Phi \mu$ is continuous too.

Also for $\sigma \in \widehat{G},(\Phi \mu) \hat{(} \sigma)=\hat{\mu}(\lambda+\delta) I_{d_{\sigma}}$ where $e^{i \lambda}, \lambda \in \underline{\underline{t}}^{*}$ is the highest weight of $\sigma[2]$.

\section{THE MAIN THEOREM}

In this section we state the main result (Theorem 3.1) of this paper and prove it.

Theorem 3.1. Let $G$ be a compact connected group or a compact Lie group. Then there exists a central, singular, continuous measure $\mu \in M(G)$ such that $\mu * \mu$ is absolutely continuous and the Lebesgue-Radon-Nikodym derivative of $\mu * \mu$ is in $L^{p}(G)$ for all $p, 1 \leq p<\infty$.

To prove Theorem 3.1, we will show that for $G$ satisfying the hypothesis of Theorem 3.1, there exists a central, singular, continuous measure $\mu$ defined on $G$ whose Fourier-Stieltjes transform $\hat{\mu}$ is $p$-summable for every $p>2$. Then $\mu * \mu$ is absolutely continuous and the Lebesgue-Radon-Nikodym derivative of $\mu * \mu$ is in $L^{p}$ for all $p, 1 \leq p<\infty$ by the Hausdorff-Young theorem ([4], Theorem 31.24). 
Proof of Theorem 3.1 for $G$ compact simply connected semisimple Lie group. Let $\{\gamma(n)\}_{n=1}^{\infty}$ be an even sequence of non-negative numbers increasing to infinity. Let $\mu$ be a non-negative continuous, singular measure on the circle group $\mathbb{T}$ such that [13]

$$
\hat{\mu}(n) \approx 0\left(\frac{\gamma(n)}{n^{1 / 2}}\right) \quad \text { and } \quad \mu(\mathbb{T})=1
$$

We define a compactly supported measure $\nu$ on $\underline{\underline{t}}$ as follows: Let $f \in C(\underline{\underline{t}})$,

$$
\langle\nu, f\rangle=\int_{0}^{2 \pi} \ldots \int_{0}^{2 \pi} \bar{f}\left(a_{1}, \ldots, a_{\ell}\right) \bar{\pi}\left(a_{1}, \ldots, a_{\ell}\right) d \mu\left(a_{1}\right) \ldots d \mu\left(a_{\ell}\right)
$$

where

$$
\begin{aligned}
& \bar{f}\left(a_{1}, \ldots, a_{\ell}\right)=f\left(H_{p}\right) \\
& \bar{\pi}\left(a_{1}, \ldots, a_{\ell}\right)=\prod_{\alpha \in R_{+}} \tilde{\alpha}\left(H_{p}\right) \text { when } p=2 a_{1} \check{\alpha}_{1}+\ldots+2 a_{\ell} \check{\alpha}_{\ell} .
\end{aligned}
$$

Let $\wedge=\left\{H_{p} \mid p=2 a_{1} \check{\alpha}_{1}+\ldots+2 a_{\ell} \check{\alpha}_{\ell}, 0 \leq a_{i} \leq 2 \pi, i=1, \ldots, \ell\right\}$. Then $\nu$ is a singular measure on $\underline{\underline{t}}$ supported in $\wedge$.

Next we define an Ad-invariant measure $\mu_{g}$ on $\underline{\underline{g}}$. Let $f \in C_{0}(\underline{\underline{g}})$,

$$
\left\langle\mu_{g}, f\right\rangle=\int_{\underline{\underline{t}}} \int_{\mathcal{O}_{H}} f(Y) d \beta_{H}(Y) d \nu(H),
$$

where $\mathcal{O}_{H}$ denotes the orbit of $H$ in $\underline{\underline{g}}$ and $\beta_{H}$ is the surface measure on $\mathcal{O}_{H}$. Then $\mu_{g}$ is a continuous, singular, Ad-invariant measure of compact support on $\underline{\underline{g}}$. Let

$$
\mu_{G}=\Phi \mu_{g}
$$

It is easily seen that $\mu_{G}$ is a continuous, central, singular measure on $G$. We show that $\left\|\hat{\mu}_{G}\right\|_{p}<\infty$ for $p>2$. Let $\sigma \in \hat{G}$; then $\hat{\mu}_{G}(\sigma)=\hat{\mu}_{g}(\lambda+\delta) I_{d_{\sigma}}$ where $\lambda$ is the highest weight for the representation $\sigma$.

Now

$$
\hat{\mu}_{g}(\lambda+\delta)=\int_{\wedge}\left(\int_{\mathcal{O}_{H}} e^{-i(\lambda+\delta)(X)} d \beta_{H}(X)\right) d \nu(H) .
$$

Using Harish-Chandra's formula for the Fourier-Stieltjes transform of a surface measure [1], we have

$$
\begin{aligned}
& \hat{\mu}_{g}(\lambda+\delta)=\frac{1}{\pi\left(H_{\lambda+\delta}\right)} \int_{\wedge} \frac{1}{\pi(H)} \sum_{w \in W} \operatorname{sgn}(w) e^{i(w \cdot(\lambda+\delta))(H)} d \nu(H) \\
& =\frac{1}{\pi\left(H_{\lambda+\delta}\right)} \sum_{w \in W} \operatorname{sgn}(w) \int_{0}^{2 \pi} \cdots \int_{0}^{2 \pi} e^{i\left(w(\lambda+\delta), 2 a_{1} \check{\alpha}_{1}+\ldots+2 a_{\ell} \check{\alpha}_{\ell}\right)} d \mu\left(a_{1}\right) \ldots d \mu\left(a_{\ell}\right) . \\
& =\frac{1}{\pi\left(H_{\lambda+\delta}\right)} \sum_{w \in W} \operatorname{sgn}(w) \prod_{i=1}^{\ell} \hat{\mu}\left(2\left\langle\lambda+\delta, w^{-1} \tilde{\alpha}_{i}\right\rangle\right) .
\end{aligned}
$$

Hence

$$
\left\|\hat{\mu}_{G}\right\|_{p}^{p}=\sum_{\sigma \in \hat{G}} d_{\sigma}\left\|\hat{\mu}_{G}(\sigma)\right\|_{p}^{p}=\sum_{\sigma \in \hat{G}} d_{\sigma}^{2}\left|\hat{\mu}_{g}(\lambda+\delta)\right|^{p}
$$


By Weyl's dimension formula, $d_{\sigma}=\frac{\prod_{\alpha \in R_{+}}\langle\lambda+\delta, \tilde{\alpha}\rangle}{\prod_{\alpha \in R_{+}}\langle\delta, \tilde{\alpha}\rangle}$, thus we obtain

$$
\left\|\hat{\mu}_{G}\right\|_{p}^{p} \leq C \sum_{\sigma \in \hat{G}} \frac{1}{\left(\prod_{\alpha \in R_{+}}\langle\lambda+\delta, \tilde{\alpha}\rangle\right)^{p-2}}\left|\sum_{w \in W} \operatorname{sgn}(w) a_{w}\right|^{p}
$$

where

$$
a_{w}=\frac{\prod_{i=1}^{\ell} \gamma\left(2\left\langle\lambda+\delta, w^{-1} \tilde{\alpha}_{i}\right\rangle\right)}{\prod_{i=1}^{\ell}\left(2\left\langle\lambda+\delta, w^{-1} \tilde{\alpha}_{i}\right\rangle\right)^{1 / 2}}, w \in W
$$

Since $G$ is simply connected, $\hat{G}$ is in one to one correspondence with the dominant integral weights given by

$$
\lambda=\sum_{i=1}^{\ell} m_{i} \lambda_{i}, \quad m_{i} \in \mathbb{Z}^{+} .
$$

Here, $\left\{\lambda_{1}, \ldots, \lambda_{\ell}\right\} \subseteq \underline{\underline{t}}^{*}$ is the set of fundamental dominant weights, i.e. $\left\langle\lambda_{i}, \tilde{\alpha}_{j}\right\rangle=$ $\delta_{i j}$. Fix $\lambda \in \hat{G}$. Suppose $\lambda=\sum_{i=1}^{\ell} m_{i} \lambda_{i}, m_{i} \in \mathbb{Z}^{+}$and $m=\max _{\alpha \in R} 2|\langle\lambda+\delta, \tilde{\alpha}\rangle|$.

Then $m \leq C_{1}\left(m_{1}+\ldots+m_{\ell}\right)$. Also for $w \in W, \prod_{i=1}^{\ell} 2\left|\left\langle\lambda+\delta, w^{-1} \tilde{\alpha}_{i}\right\rangle\right| \geq$ $\prod_{i=1}^{\ell}\left(m_{i}+1\right)$. Hence

$$
\begin{aligned}
\left\|\hat{\mu}_{G}\right\|_{p}^{p} & \leq C \sum_{m_{1}, \ldots, m_{\ell} \in \mathbb{Z}^{+}} \frac{(\gamma(m))^{p \ell}|W|^{p}}{\left(\prod_{i=1}^{\ell}\left\langle\lambda+\delta, \tilde{\alpha}_{i}\right\rangle\right)^{p-2} \prod_{i=1}^{\ell}\left(m_{i}+1\right)^{p / 2}} \\
& \leq C \sum_{m_{1}, \ldots, m_{\ell} \in \mathbb{Z}^{+}} \frac{(\gamma(m))^{p \ell}|W|^{p}}{\prod_{i=1}^{\ell}\left(m_{i}+1\right)^{\frac{3 p-4}{2}}} .
\end{aligned}
$$

Let

$$
\begin{aligned}
\gamma(n) & =0 \quad \text { if }|n| \leq 1 \\
& =\log |n| \quad \text { if }|n|>1 .
\end{aligned}
$$

Then

$$
\begin{aligned}
\left\|\hat{\mu}_{G}\right\|_{p}^{p} & \leq C|W|^{p} \sum_{m_{1}, \ldots, m_{\ell} \in \mathbb{Z}^{+}} \frac{\left(\log C_{1}+\prod_{i=1}^{\ell} \log \left(m_{i}+1\right)\right)^{p \ell}}{\prod_{i=1}^{\ell}\left(m_{i}+1\right)^{\frac{3 p-4}{2}}} \\
& \leq C_{2} \sum_{m_{1}, \ldots, m_{\ell} \in \mathbb{Z}^{+}} \frac{\left(\prod_{i=1}^{\ell} \log \left(m_{i}+1\right)\right)^{p \ell}}{\prod_{i=1}^{\ell}\left(m_{i}+1\right)^{\frac{3 p-4}{2}}} \\
& =C_{2} \prod_{i=1}^{\ell} \sum_{m=0}^{\infty} \frac{(\log (m+1))^{p \ell}}{(m+1)^{\frac{3 p-4}{2}}}<\infty \text { for } p>2 .
\end{aligned}
$$

The proof is complete.

To complete the proof of Theorem 3.1 in the general case, we first prove two lemmas. For convenience, we will say that the group $G$ has property $\underline{P}$ if there exists a continuous, singular, central measure on $G$ whose Fourier-Stieltjes transform is $p$-summable for every $p>2$. 
Lemma 3.2. Let $T$ be a compact connected abelian group, $\left\{G_{\alpha}\right\}_{\alpha \in I}$ a family of compact, simply connected, simple Lie groups and $K$ a totally disconnected closed subgroup of the centre of $T \times \prod_{\alpha \in I} G_{\alpha}$. Suppose that there exists a $\beta \in I$ such that $G_{\beta}$ has property $\underline{P}$. Then

$$
G=T \times \prod_{\alpha \in I} G_{\alpha} / K
$$

has property $\underline{P}$.

Proof. Let $\mu_{\beta}$ be a measure on $G_{\beta}$ corresponding to property $\underline{P}$ of $G_{\beta}$. Define $\nu=\prod_{\alpha \in(I-\{\beta\})} \mu_{\alpha} \times \mu_{\beta}$, where $\mu_{\alpha}$ is Haar measure on $G_{\alpha}$ for $\alpha \in(I-\{\beta\})$.

Then $\nu$ is a continuous, central, singular measure on $T \times \prod_{\alpha \in I} G_{\alpha}$ and the measure $\mu$ induced by $\nu$ on $G$ shows that $G$ has property $\underline{P}$. This completes the proof.

Lemma 3.3. Let $G$ be any compact group. Let $G_{0}$ be an open normal subgroup of $G$ satisfying the property $\underline{P}$. Then $G$ satisfies $\underline{P}$.

Proof. Define a measure $\mu$ on $G$ as follows: Let $E$ be a Borel measurable subset of $G$,

$$
\mu(E)=\mu_{0}\left(E \cap G_{0}\right),
$$

when $\mu_{0}$ is a measure on $G_{0}$ satisfying $\underline{P}$. It is clear that $\mu$ is central, singular, continuous measure on $G$. We show that $\|\hat{\mu}\|_{p}<\infty$ for every $p>2$.

Let $n$ be the index of $G_{0}$ in $G$. If $\sigma \in \hat{G}$, then by Clifford's theorem ([8], Theorem 12.1) there exists $\alpha \in \hat{G}_{0}$ and natural numbers $k, n_{\sigma}\left(k, n_{\sigma} \leq n\right)$ such that

$$
\begin{aligned}
\left.\sigma\right|_{G_{0}} & =n_{\sigma}\left(\alpha^{\left(g_{1}\right)}+\ldots+\alpha^{\left(g_{k}\right)}\right) \quad \text { when } \\
\alpha^{(g)}(h) & =\alpha\left(g^{-1} h g\right), \quad g \in G, h \in G_{0},
\end{aligned}
$$

and $\left\{e=g_{1}, g_{2}, \ldots, g_{k}\right\}$ is a complete set of coset representatives for $H_{\alpha}=\{g \in$ $G \mid \alpha^{(g)}$ is equivalent to $\left.\alpha\right\}$.

Let $\hat{\mu}(\sigma)=c_{\sigma} I_{d_{\sigma}}$. Now

$$
\begin{aligned}
c_{\sigma} & =\frac{1}{d_{\sigma}} \int_{G} \chi_{\sigma}\left(x^{-1}\right) d \mu(x) \\
& =\frac{n_{\sigma} d_{\alpha}}{d_{\sigma}}\left(c_{\alpha^{\left(g_{1}\right)}}+\ldots+c_{\alpha^{\left(g_{k}\right)}}\right) \text { where } \hat{\mu}_{0}\left(\alpha^{\left(g_{i}\right)}\right)=c_{\alpha^{\left(g_{i}\right)}} I_{d_{\alpha}} .
\end{aligned}
$$

Let $p>2$. Then

$$
\begin{aligned}
\|\hat{\mu}\|_{p}^{p} & =\sum_{\sigma \in \hat{G}} d_{\sigma}\|\hat{\mu}(\sigma)\|_{p}^{p}=\sum_{\sigma \in \hat{G}} d_{\sigma}^{2}\left|c_{\sigma}\right|^{p} \\
& =\sum_{\sigma \in \hat{G}} d_{\sigma}^{2}\left(\frac{n_{\sigma} d_{\alpha}}{d_{\sigma}}\right)^{p}\left(\sum_{i=1}^{k}\left|c_{\alpha^{\left(g_{i}\right)}}\right|\right)^{p} .
\end{aligned}
$$

Since $d_{\sigma}=d_{\alpha} n_{\sigma} k$, we get

$$
\|\hat{\mu}\|_{p}^{p}=\sum_{\sigma \in \hat{G}} \frac{d_{\alpha}^{2} n_{\sigma}^{2}}{k^{p-2}}\left(\sum_{i=1}^{k}\left|c_{\alpha^{\left(g_{i}\right)}}\right|\right)^{p} .
$$


Since ' $\alpha$ ' can occur in at most $n$ irreducible representations of $G$ (as $\left.1 \leq n_{\sigma} \leq n\right)$, we get

$$
\begin{aligned}
\|\hat{\mu}\|_{p}^{p} & \leq n \sum_{\alpha \in \hat{G}_{0}} d_{\alpha}^{2} n_{\sigma}^{2}\left(\sum_{i=1}^{k}\left|c_{\alpha\left(g_{i}\right)}\right|\right)^{p} \\
& \leq n^{4} \sum_{\alpha \in \hat{G}_{0}} d_{\alpha}^{2} \max _{1 \leq i \leq k}\left|c_{\alpha^{\left(g_{i}\right)}}\right|^{p} .
\end{aligned}
$$

Now $\sum_{\alpha \in \hat{G}_{0}} d_{\alpha}^{2} \max _{1 \leq i \leq k}\left|c_{\alpha^{\left(g_{i}\right)}}\right|^{p} \leq \sum_{\alpha \in \hat{G}_{0}} d_{\alpha}^{2}\left|c_{\alpha}\right|^{p}$. Therefore

$$
\|\hat{\mu}\|_{p}^{p} \leq n^{4} \sum_{\alpha \in \hat{G}_{0}} d_{\alpha}^{2}\left|c_{\alpha}\right|^{p}=n^{4}\left\|\hat{\mu}_{0}\right\|_{p}^{p}<\infty
$$

for $p>2$.

This completes the proof.

End of the proof of Theorem 3.1. The theorem follows now by a standard argument using the structure theorem of compact connected groups (see [9]), Lemma 3.2 and Lemma 3.3.

Remark 3.4. S. Sakai [12] has shown that there exists a singular probability measure $\mu$ on the circle group $\mathbb{T}$ such that $\mu * \mu$ is absolutely continuous and the RadonNikodym derivative of $\mu * \mu$ has a uniformly convergent Fourier series. We are unable to show that the measure $\mu$ constructed in Theorem 3.1 has this stronger property.

\section{TOtALly DisCONNECTED GROUPS}

In this section we prove the following analogue of Theorem 3.1 on some totally disconnected groups.

Theorem 4.1. Let $\left\{G_{n}\right\}_{n=1}^{\infty}$ be a sequence of finite non-abelian groups such that there exist two constants $C_{1}, C_{2}>0$ satisfying: For every $n$, there exists an irreducible character $\chi_{n}$ of $G_{n}$ such that

$$
\begin{gathered}
\operatorname{Re}\left(\chi_{n}\right) \geq-C_{1} \quad \text { on } G_{n} \\
\text { and } \quad \frac{1}{\left|G_{n}\right|} \sum_{x \in A_{n}}\left(\operatorname{Re} \chi_{n}(x)\right)^{2} \geq C_{2} \\
\text { when } A_{n}=\left\{x \in G_{n} \mid \operatorname{Re} \chi_{n}(x)<0\right\} .
\end{gathered}
$$

Then there exists a continuous, singular, central measure $\mu$ on $G=\prod_{n=1}^{\infty} G_{n}$ such that $\mu * \mu$ is absolutely continuous with respect to Haar measure on $G$ and the Lebesgue Radon-Nikodym derivative of $\mu * \mu$ belongs to $L^{p}$ for all $p, 1 \leq p<\infty$.

Proof. Let $\left\{b_{n}\right\}_{n=1}^{\infty}$ be a sequence of non-negative numbers such that

$$
0<b_{n} C_{1}<1 \text { for every } n, \quad \sum_{n=1}^{\infty} b_{n}^{2}=\infty \text { and } \sum_{n=1}^{\infty} b_{n}^{p}<\infty, \text { for every } p>2 .
$$

Define $g_{n}=\operatorname{Re} \chi_{n}$, and $f_{n}=1+b_{n} g_{n}$. 
Let $\mu=\prod_{n=1}^{\infty} f_{n} d x$, where $d x$ denotes the Haar measure on $G_{n}$. Then $\mu$ is a central continuous measure on $G$.

$\mu$ is singular: By Kakutani's criterion ([5], Theorem 22.36) for singularity of product measures, it is sufficient to show that

$$
\prod_{n=1}^{\infty} \int_{G_{n}} f_{n}^{1 / 2} d x=0
$$

Now

$$
\int_{G_{n}} f_{n}^{1 / 2} d x=\int_{A_{n}} f_{n}^{1 / 2} d x+\int_{G_{n}-A_{n}} f_{n}^{1 / 2} d x
$$

Also

$$
\begin{aligned}
\int_{A_{n}} f_{n}^{1 / 2} d x & =\frac{1}{\left|G_{n}\right|} \sum_{x \in A_{n}}\left(1-\left(-b_{n} g_{n}\right)(x)\right)^{1 / 2} \\
& \leq \frac{1}{\left|G_{n}\right|} \sum_{x \in A_{n}}\left(1+\frac{b_{n} g_{n}(x)}{2}-\frac{b_{n}^{2}}{16} g_{n}^{2}(x)\right)
\end{aligned}
$$

and

$$
\begin{aligned}
\int_{G_{n}-A_{n}} f_{n}^{1 / 2} d x & \leq\left(\int_{G_{n}-A_{n}} f_{n} d x\right)^{1 / 2}\left(\frac{\left|G_{n}\right|-\left|A_{n}\right|}{\left|G_{n}\right|}\right)^{1 / 2} \\
& \leq \frac{\left(\left|G_{n}\right|-\left|A_{n}\right|\right)}{\left|G_{n}\right|}\left(1+\frac{\sum_{x \in A_{n}}\left(-b_{n} g_{n}(x)\right)}{2\left(\left|G_{n}\right|-\left|A_{n}\right|\right)}\right) .
\end{aligned}
$$

By (4.4) and (4.5), we get

$$
\int_{G_{n}} f_{n}^{1 / 2} d x \leq 1-\frac{1}{\left|G_{n}\right|} \sum_{x \in A_{n}} \frac{b_{n}^{2} g_{n}^{2}(x)}{16} \leq 1-\frac{b_{n}^{2} C_{2}}{16} .
$$

Since $\sum_{n=1}^{\infty} b_{n}^{2}=\infty$, we conclude ([5], Lemma 22.25) that $\prod_{n=1}^{\infty} \int_{G_{n}} f_{n}^{1 / 2}=0$.

Computation of $\|\hat{\mu}\|_{p}$ : Let $p>2$.

$$
\begin{aligned}
\|\hat{\mu}\|_{p}^{p} & =\sum_{\sigma \in \hat{G}} d_{\sigma}\|\hat{\mu}(\sigma)\|_{p}^{p}=\sum_{\sigma \in \hat{G}} d_{\sigma}^{2-p} \prod_{i=1}^{k}\left|\left(\frac{b_{n_{i}}}{2}\left(1+\int_{G_{n_{i}}}{\overline{\chi_{n_{i}}(x)}}^{2} d x\right)\right)\right|^{p} \\
& \leq \sum_{n_{1}, \ldots, n_{k} \in \mathbb{N}} \prod_{i=1}^{k}\left(b_{n_{i}}^{p}\right)=\prod_{i=1}^{\infty}\left(1+b_{i}^{p}\right)<\infty \text { as } \sum_{i=1}^{\infty} b_{i}^{p}<\infty .
\end{aligned}
$$

This completes the proof of the theorem.

Remark 1. Conditions (4.2) and (4.3) are satisfied by $G=\prod_{n=1}^{\infty} G_{n}$ if $G_{n}$ 's are symmetric groups, dihedral groups and generalised quaternion groups (see [4], 27.61).

\section{REFERENCES}

1. J.L. Clerc, Orbites dans le plan tangent d'un espace symétrique, mesures orbitales et leurs transformées de Fourier, Topics in Modern Harmonic Analysis, Vol.I, Proc. of a seminar held in Torino and Milano, May-June 1982, pp. 259-300. MR 86c:22013

2. A.H. Dooley and N.J. Wildberger, Global formulae on compact Lie groups, Funktsional. Anal. i Prilozhen. 27 (1) (1993), 25-32; English transl., Functional Anal. Appl. 27 (1993), 21-27. MR 94e:22032

3. E. Hewitt and K. Ross, Abstract Harmonic Analysis, Vol.I, Springer-Verlag, Berlin, 1963. MR 28:158 
4. E. Hewitt and K. Ross, Abstract Harmonic Analysis, Vol.II, Springer-Verlag, Berlin, 1970. MR 42:7378

5. E. Hewitt and K. Stromberg, Real and Abstract Analysis, 3rd printing, Springer-Verlag, Berlin, 1975. MR 51:3363

6. E. Hewitt and H. Zuckerman, Singular measures with absolutely continuous convolution squares, Proc. Cambridge Phil. Soc. 62 (1966), 399-420; , vol. 63, 1967, pp. 367-368. MR 33:1655; MR 34:8497

7. C. Karanikas and S. Koumandos, Continuous singular measures with absolutely continuous convolution squares and locally compact groups, Illinois J. of Math. 35 (3) (1991), 490-495. MR 92b: 43004

8. G.W. Mackey, Induced representations of locally compact groups I, Ann. of Math. 55 (1952), 101-139. MR 13:434

9. J.F. Price, Lie groups and compact groups, Cambridge University Press, Cambridge, 1977. MR 56:8743

10. D.L. Ragozin, Central measures on compact simple Lie groups, J. of Functional Analysis 10 (1972), 212-229. MR 49:5715

11. S. Saeki, Singular measures having absolutely continuous convolution powers, Illinois J. Math. 21 (1977), 395-412. MR 58:6719

12. S. Saeki, On convolution squares of singular measures, Illinois J. Math. 24 (1980), 225-232. MR 81i: 42005

13. A.C. Schaeffer, The Fourier-Stieltjes coefficients of a function of bounded variation, Amer. J. Math. 61 (1939), 934-940. MR 1:12

14. K. Stempak, On convolution products of radial measures on the Heisenberg group, Colloq. Math. 50 (1985), 125-128. MR 87d:22014

School of Mathematics, University of New South Wales, New South Wales 2052, Australia

E-mail address: tony@solution.maths.unsw.edu.au

E-mail address: sanjiv@solution.maths.unsw.edu.au

Current address, S. Gupta: Department of Mathematics, University of South Pacific, Suva, Fiji Islands 